\title{
Use of High-Resolution Ultrasound (HRU) in the Assessment of Deep Injections of CHAP-Hyaluronic Acid (CHAP-HA) Fillers for Midface Lift
}

\author{
Hsiao-Tung Lee1, Haw-Yueh Thong2* \\ ${ }^{1}$ Department of Radiology, Shin-Kong Wu Ho-Su Memorial Hospital, Taiwan \\ ${ }^{2}$ Department of Dermatology, Shin-Kong Wu Ho-Su Memorial Hospital, Taiwan \\ Email: *drkellytang@gmail.com
}

How to cite this paper: Lee, H.-T. and Thong, H.-Y. (2018) Use of High-Resolution Ultrasound (HRU) in the Assessment of Deep Injections of CHAP-Hyaluronic Acid (CHAPHA) Fillers for Midface Lift. Journal of Cosmetics, Dermatological Sciences and Applications, 8, 126-132.

https://doi.org/10.4236/jcdsa.2018.83014

Received: May 22, 2018

Accepted: September 10, 2018

Published: September 13, 2018

Copyright $\odot 2018$ by authors and Scientific Research Publishing Inc. This work is licensed under the Creative Commons Attribution International License (CC BY 4.0).

http://creativecommons.org/licenses/by/4.0/

\begin{abstract}
High-resolution ultrasound (HRU) imaging is a useful tool to study hyaluronic acid (HA) filler injection in the face. It is noninvasive, quick, well-tolerated, and can provide in vivo and dynamic information. The formations of pools or pearls in HA fillers could be observed real time during injection. The plane of injection could be determined accurately, and there were no specimen manipulation artifacts. It was observed that HA gel fillers with differing production technologies showed distinct spread and distribution patterns in the periocular tissues on HRU examination. The authors used HRU to assess deep injections of CHAP-Hyaluronic Acid (CHAP-HA) fillers for midface lift. 10 patients who underwent bilateral midface deep injections using CHAP-HA filler were examined with HRU before and immediately after treatment, and in 2 weeks and one month later. The CHAP-HA appeared as hypoechoic densities within the preperiosteal plane in HRU. CHAP-HA adopted variable morphology within the tissue depending on individual tissue densities and the compliance of the tissues in the plane of injection. CHAP-HA was unidentifiable with surrounding tissue after one month in 13 of the 20 injection sites. HRU allows in vivo study of CHAP-HA injection behavior and could be a tool for further studies of HA-tissue reactions.
\end{abstract}

\section{Keywords}

CHAP-Hyaluronic Acid (CHAP-HA) Filler, High-Resolution Ultrasound (HRU), Midface Lift, Deep Injections, Preperiosteal Filler Injections 


\section{Introduction}

High-resolution ultrasound (HRU) made the visualization of hyaluronic acid (HA) filler possible within the facial soft tissues, providing perspectives on the shape, size, and location of the HA when injected [1]-[6]. It could also be a useful tool to study HA-tissue interactions and is generally well-tolerated. This may be especially helpful during periorbital injections, where thin skin overlies bone and the complex three-dimensional contours are particularly challenging [7].

Most HRU imagings of HA injections typically showed consistent and characteristic "black pearls" in the predicted depth, in the areas where volume had been restored clinically [8]. However, Goh et al. reported that HA gel fillers with differing production technologies showed distinct spreads and distribution patterns in the periocular tissues on HRU examination [9]. Such variability could be the results of the interactions between the injection techniques, HA particles, plane of injections, and individual tissue compliance.

\section{Aim}

The purpose of this pilot observational study was to observe injections of CHAP-Hyaluronic acid (CHAP-HA) by using HRU to have a better understanding of the behavior of CHAP-HA after being injected deeply into preperiosteal area, and the chronological changes.

\section{Methods}

\subsection{Material}

The CHAP-HA fillers, HYADERMIS ${ }^{\text {ma }}$ product line (SciVision Biotech Inc., Taiwan), was approved by Taiwan FDA in 2010 and consists of HYADERMIS

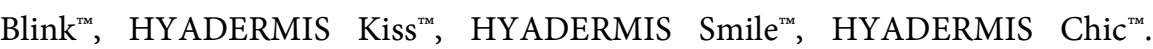
HYADERMIS $^{\mathrm{TM}}$ is composed of bacterium-derived non-animal stabilized HA with a homologous particle consistency that uses a crosslinked technology called

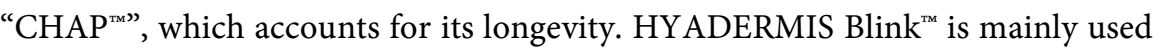
to improve fine lines and as skin boosters, HYADERMIS ${ }^{\text {mi }}$ Kiss is used for correcting tear trough and for lip augmentation; whereas HYADERMIS ${ }^{\text {Tx }}$ Smile and HYADERMIS $^{\text {tm }}$ Chic are best for contouring, volumizing medium depth facial wrinkles and for lifting. HYADERMIS ${ }^{\mathrm{Tm}}$ is one of the commonly used dermal filler in Taiwan. In our study, 2 syringes of $1 \mathrm{ml}$-HYADERMIS Smile ${ }^{\text {Tx }}$ were used ( $1 \mathrm{ml}$ each for each side of the face) for cheek augmentation in each subject.

\subsection{Subjects}

Ten healthy non-pregnant female individuals with mild to moderate midface ptosis, who have never received dermal filler injection were enrolled and received single-point deep injection of HYADERMIS Smile ${ }^{\text {tm }}$ at midface. Treatment area with tattoos, scars, dermatitis, or open wounds, and individuals with a history of major diseases, diabetes mellitus, HIV infection, connective tissue diseases, and malignant diseases were excluded. Those with recent (within 3 
months) aesthetic laser/chemical peeling treatment and concurrent use of medication that could disrupt coagulation (e.g., aspirin, NSAIDs, warfarin) were also excluded. All subjects provided written informed consent.

\subsection{Preparation and Treatment}

Treatment areas were cleansed with a mild cleanser. All patients were photographed prior to injection. Topical anesthetics were applied for 10 minutes. Prior to injection, HRU examination was performed (iU22, Philips) to briefly evaluate the presence of blood vessels on the intended injection point. After the confirmation of an absence of major blood vessels, single point injection of $1 \mathrm{ml}$ CHAP-HA was performed on each midcheek using either sharp needle or micro-cannula, depending on the preference of the subjects (Figure 1).

Before and after injection, ultrasound imaging was performed to confirm the depth of injection (Figure 2).

HRU visualization (iU22, Philips) was performed before and immediately after HA filler injection by a single operator, using a $12-5 \mathrm{MHz}$ linear transducer. Images showing the location, size, and shape of the HA after injection, and at 2-week and 4-week follow-up were obtained for analysis. Features related to the shape and tissue distribution of HA gel filler were defined. Relationships to regional bony and soft tissue structures were also recorded.

\section{Results}

10 female subjects completed the treatments. All subjects were Taiwanese, with a mean age of 39.4 years (whose ages ranged from 28 to 50 years old). All subjects had mild to moderate midface ptosis. All subjects received a single-point injection of $1 \mathrm{ml}$ CHAP-HA (HYADERMIS ${ }^{\mathrm{TM}}$ Smile) on each side of the midface region. 6 subjects chose to be injected with sharp needle, whereas 4 subjects decided to be injected with microcannula. Total of 20 injection sites were evaluated with HRU. In all sites, the CHAP-HA particles appeared as hypoechoic densities within the soft tissue plane, with some particles spreading into bubbles or pearl-like configurations (Figure 3 and Figure 4). Depending on the extent of spread and the surrounding tissue compliance, some variabilities could occur, showing areas of mixed hypoechoic and hyperechoic HA imaging findings (Figure 4(d) and Figure 4(f)).

There was no clinically significant difference between sharp needle and microcannula injections on cheek augmentation effect and degree of edema. There was also no difference in the HRU imaging between sharp needle versus microcannula injections in terms of the morphology, shape and size of the CHAP-HA after injection.

Follow-up HRU imaging at 2 and 4 weeks showed that hydration of the CHAP-HA would occur, and the CHAP-HA would appear to be more heterogenous and hyperechoic (Figure 5). In the majority of cases (13 of the 20 injections in 8 patients), the HA became completely unidentifiable with the surrounding 


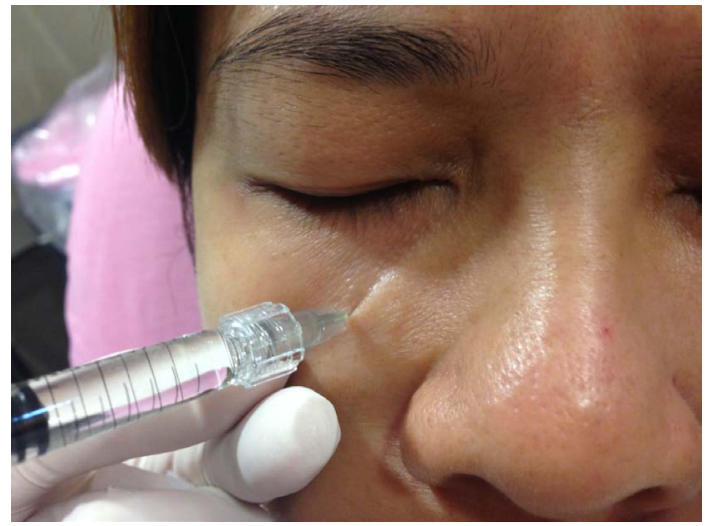

Figure 1. Single point injection of $1 \mathrm{ml}$ CHAP-HA was performed on midcheek using either sharp needle.

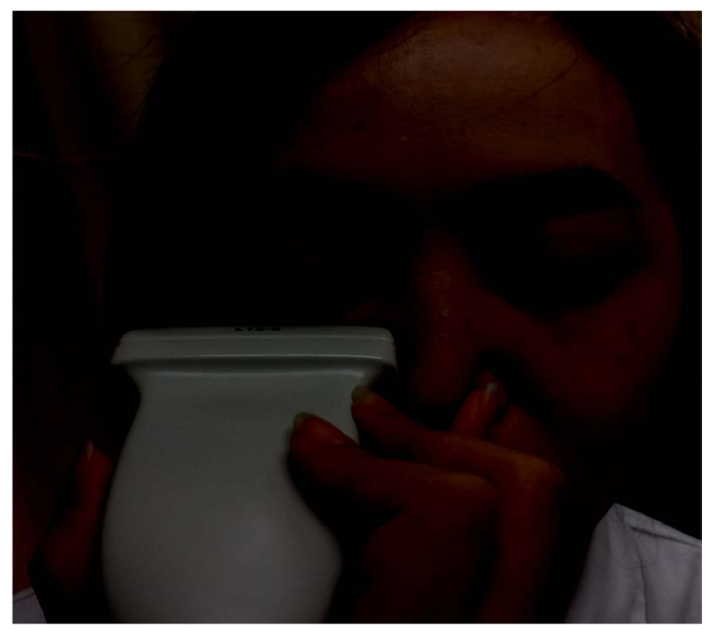

Figure 2. High-resolution ultrasound imaging was performed using a $12-5 \mathrm{MHz}$ linear transducer to see the location, size, and shape of the ha on horizontal section.

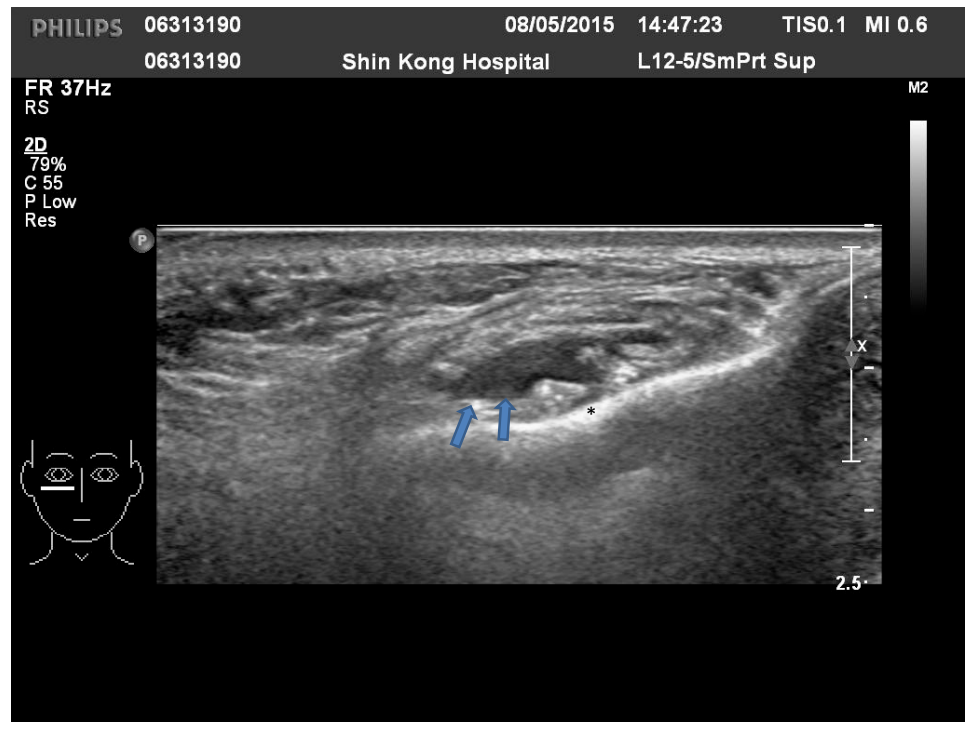

Figure 3. Ultrasound imaging of HA filler after injection to confirm depth of filler placement. arrow $=$ HA filler, ${ }^{*}=$ periosteum. 


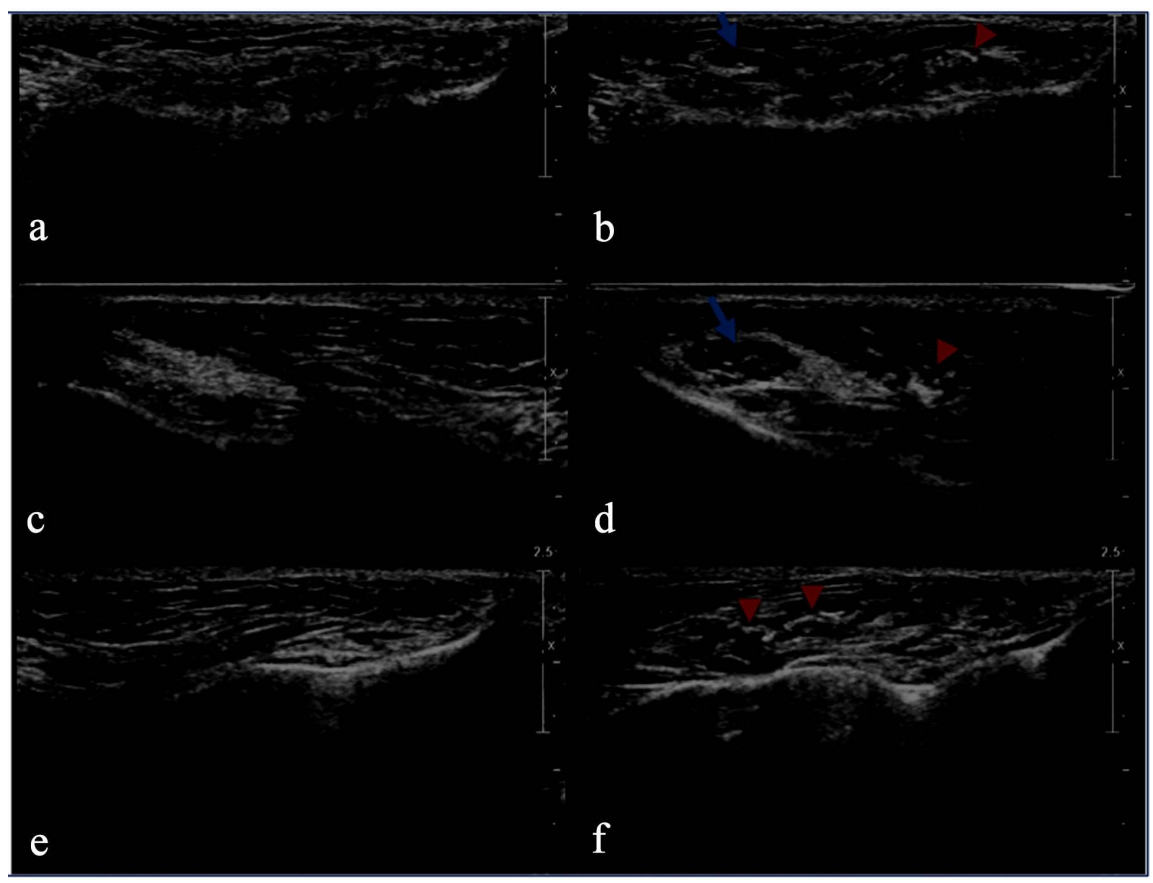

Figure 4. High-resolution ultrasound imaging performed before (a, c, e) and immediately after ( $\mathrm{b} \mathrm{d} \mathrm{f)} \mathrm{HA} \mathrm{filler} \mathrm{injection.} \mathrm{The} \mathrm{HA} \mathrm{filler} \mathrm{formed} \mathrm{a} \mathrm{localized} \mathrm{hypoechoic} \mathrm{area} \mathrm{within}$ the tissue (arrows), some spread into hypoechoic bubbles or pearls and mixed with hyperechogenicity (arrowheads).

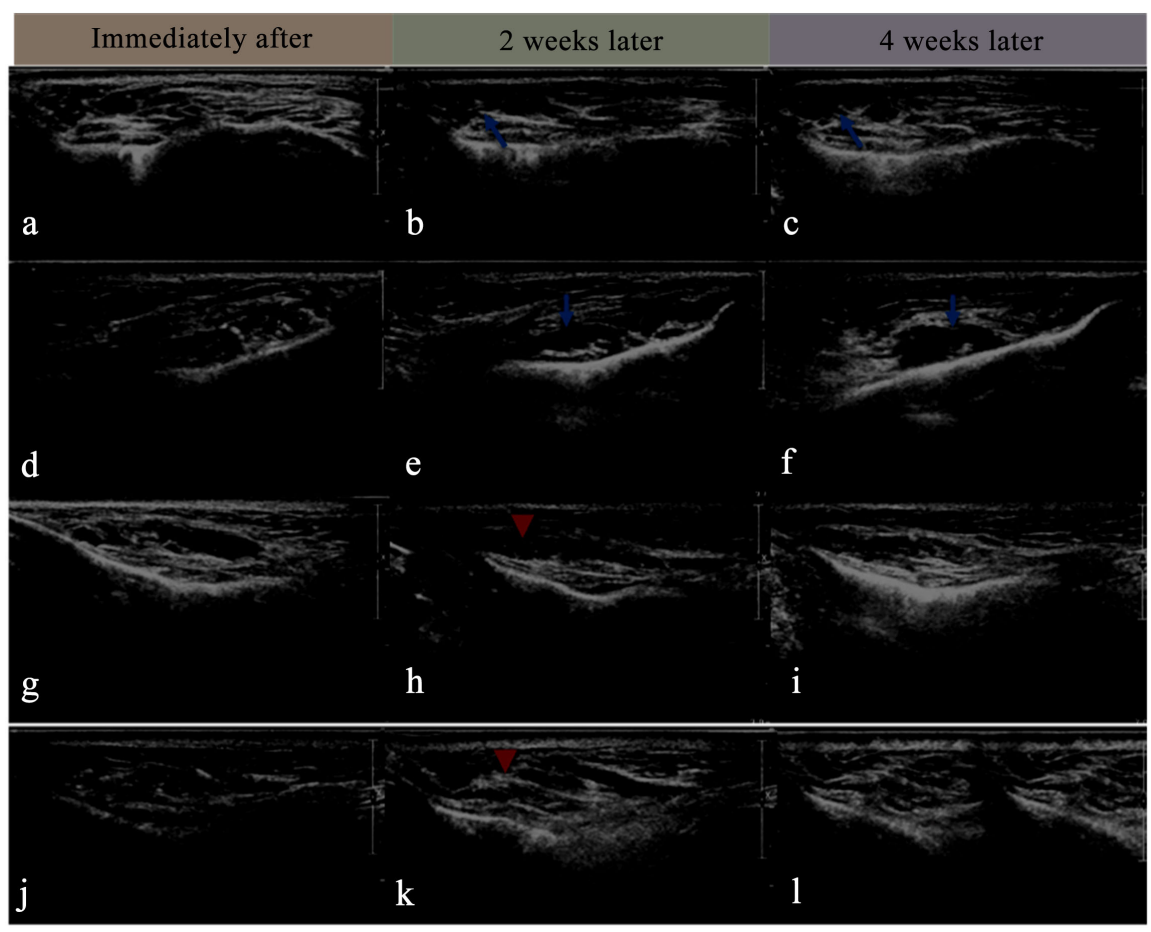

Figure 5. High-resolution ultrasound imaging immediately after HA injection (a, d, g, j), at 2-Week (d, e, h, k) and 4-week (c, f, i, 1) follow up. Hydration of the HA would occur (arrows), and the ha would appear to be more heterogenous and hyperechoic (arrowheads) and may became completely unidentifiable with the surrounding tissues in the 4 th week follow up $(i, j)$. 
tissues at the $4^{\text {th }}$ week follow-up (Figure 5(i) and Figure 5(1)).

\section{Discussion}

HRU could provide in vivo and dynamic information of the HA pearls which were injected. The plane of injection could be determined accurately. Conventional HRU imaging was captured using 5- to $15-\mathrm{MHz}$ transducers allowing visualization of 0.5 to $3 \mathrm{~cm}$ deep through the skin. The resulting images included the epidermis (thickness $0.06-0.6 \mathrm{~mm}$ ), dermis (thickness $1-4 \mathrm{~mm}$ ), and subcutaneous tissues (thickness $5-20 \mathrm{~mm}$ ) within the best focal range [10]. The density and organization of the tissues determined the tissue appearances in B-scans (two-dimensional black-and-white images obtained by the reflection of ultrasound echo while passing through tissues), resulting in images that resemble anatomic cross-sections [11]. Sound waves passed through fluid collections and HA without reflection to the transducer, resulting in totally black or anechoic images. The opposite happened in dense tissues such as bone, in which greater reflection produced bright white or hyperechoic images. The epidermis appeared as a thin hyperechoic line, and the dermis as a slightly less bright hyperechoic band of variable thickness. The subcutaneous tissue had hypoechoic fatty lobules with hyperechoic fibrous septa in between, and the muscle fascia was observed as a hyperechoic regular line [1] [2].

Visualization of the preperiosteal HA was very challenging because the anechoic pearls or bubbles ultrasound images could look very similar to the normal muscles and fatty tissues. This was the same phenomenon that occurred in the cheek area and may represent a limitation of HRU in the study of deep HA placement [12]. This also explained some of the variable echogenicities showed in our studies, probably due to the complex hydrodynamic characteristics of CHAP-HA, plane of injections, and individual tissue densities. Kohn et al. suggested that deep injection in the preperiosteal plane may require a larger volume of HA to have better HRU visualization, because the HA would diffuse faster and further when injected [9]. The tissues provided less contrast, so more HA may be needed to obtain reliable images. Our study demonstrated that $1 \mathrm{ml}$ CHAP-HA in each side of the cheek was sufficient in most cases to obtain adequate HRU visualization.

\section{Conclusion}

Single-point deep injection of CHAP-HA filler at midface is a technique which is relatively quick and easy to perform. HRU evaluation before injection could be a helpful tool to avoid inadvertent intravascular injection and could also be used to confirm the depth of injection. HRU could also serve as a reliable tool to study HA-tissue interactions. HA fillers with different formulations and dilutions may show distinctive features after injection. Future studies should incorporate additional subjects and investigate HRU properties of other HA formulations in this area of the face. 


\section{Disclosures}

The authors have no conflict of interest to disclose.

There are no funding sources for this work.

\section{Conflicts of Interest}

The authors declare no conflicts of interest regarding the publication of this paper.

\section{References}

[1] Schelke, L.W., Van Den Elzen, H.J., Erkamp, P.P. and Neumann, H.A. (2010) Use of Ultrasound to Provide Overall Information on Facial Fillers and Surrounding Tissue. Dermatologic Surgery, 36, 1843-1851. https://doi.org/10.1111/j.1524-4725.2010.01740.x

[2] Wortsman, X. (2012) Common Applications of Dermatologic Sonography. Journal of Ultrasound in Medicine, 31, 97-111. https://doi.org/10.7863/jum.2012.31.1.97

[3] Young, S.R., Bolton, P.A. and Downie, J. (2008) Use of High-Frequency Ultrasound in the Assessment of Injectable Dermal Fillers. Skin Research and Technology, 14, 320-323. https://doi.org/10.1111/j.1600-0846.2008.00297.x

[4] Wortsman, X., Wortsman, J., Orlandi, C., Cardenas, G., et al. (2011) Ultrasound Detection and Identification of Cosmetic Fillers in the Skin. Journal of the European Academy of Dermatology and Venereology, 26, 292-301. https://doi.org/10.1111/j.1468-3083.2011.04047.x

[5] Grippaudo, F.R. and Mattei, M. (2010) High-Frequency Sonography of Temporary and Permanent Dermal Fillers. Skin Research and Technology, 16, 265-269. https://doi.org/10.1111/j.1600-0846.2010.00428.x

[6] Grippaudo, F.R. and Mattei, M. (2011) The Utility of High-Frequency Ultrasound in Dermal Filler Evaluation. Annals of Plastic Surgery, 67, 469-473. https://doi.org/10.1097/SAP.0b013e318203ebf6

[7] Goldberg, R.A. (2005) The Three Periorbital Hollows: A Paradigm for Periorbital Rejuvenation. Plastic and Reconstructive Surgery, 116, 1796-1804. https://doi.org/10.1097/01.prs.0000185623.36795.38

[8] Kohn, J.C., Goh, A.S., Lin, J.L. and Goldberg, R.A. (2013) Dynamic High-Resolution Ultrasound in Vivo Imaging of Hyaluronic Acid Filler Injection. Dermatologic Surgery, 39, 1630-1636. https://doi.org/10.1111/dsu.12345

[9] Goh, A.S., Kohn, J.C., Rootman, D.B., Lin, J.L. and Goldberg, R.A. (2014) Hyaluronic Acid Gel Distribution Pattern in Periocular Area with High-Resolution Ultrasound Imaging. Aesthetic Surgery Journal, 34, 510-515. https://doi.org/10.1177/1090820X14528206

[10] Cammarota, T.F., Magliaro, P.A. and Sarno, A. (1998) Current Uses of Diagnostic High-Frequency US in Dermatology. European Journal of Radiology, 27, S215-223. https://doi.org/10.1016/S0720-048X(98)00065-5

[11] Wendtner, S., Hildegard, M. and Burgdorf, W. (2005) Ultrasound Scanning in Dermatology. Archives of Dermatology, 141, 217-224.

[12] Papageorgiou, K., Chang, H.S.H., Isaacs, D., Fiaschetti, D., et al. (2012) Refining the Goals of Oculofacial Rejuvenation with Dynamic Ultrasonography. Aesthetic Surgery Journal, 32, 207-219. https://doi.org/10.1177/1090820X11434523 